\title{
Parametrization of the molecular free energy surface density (MolFESD) for different solvents and brain-blood barrier
} partitioning

\author{
Mohamed Zerara*1, R Kretschmer², Thomas E Exner ${ }^{2}$ and J Brickmann ${ }^{1}$
}

Address: ${ }^{1}$ MOLCAD Research Unit - Molcad GmbH and TU-Darmstadt, Petersenstr. 20, 64287 Darmstadt, Germany and ${ }^{2}$ Universität Konstanz, Fachbereich Chemie, Fach 721, 78457 Konstanz, Germany

* Corresponding author

from 3rd German Conference on Chemoinformatics

Goslar, Germany. II-13 November 2007

Published: 26 March 2008

Chemistry Central Journal 2008, 2(SuppI I):P20 doi:10.I 186/I752-I53X-2-SI-P20

This abstract is available from: http://www.journal.chemistrycentral.com/content/2/SI/P20

(c) 2008 Zerara et al.

Quantitative information of solvation and transfer free energies is often needed for the understanding of many physicochemical processes, e.g the molecular recognition phenomena, the transport and diffusion processes through biological membranes and the tertiary structure of proteins. Recently, the molecular free energy surface density concept (MolFESD) has been introduced [1,2]. This model is based on the assumptions that the overall hydrophobicity can be obtained as a superposition of fragment contributions and that the corresponding free energy can be calculated as an integral of the MolFESD over the molecular solvent accessible surface. The scalar quantity 3D-FED, the three-dimensional free energy density offers a physical basis to the establishment of a new predictive model with limited empirical character. Although this volume density is accessible from more accurate methods e.g. Monte-Carlo methods, the Grid program [3] has proved to be suitable for a rapid evaluation of the 3D-FED. It uses empirical force field to determine the interaction energy of a particular probe molecule (e.g water) for all points of a regular three dimensional grid in which the target molecule is enclosed. In the present study, parametrization of the MolFESD is done in two steps using the same strategy described in the previous work of Jäger et al. [1,2]; some model parameters were fitted to computed data (provided by Grid program) while other parameters were fitted to experimental $\log P$ values. Unlike most fragment based predictive models for $\log P$, the MolFESD concept calculates $\log P$ values which depend on the area and shape of molecular surfaces and in turn, on the corresponding molecular conformations. A parametrization of the MolFESD is done for three solvents (n-octanol/water, alkane/water and cyclohexane/water) followed by its application to the prediction of brainblood barrier partition coefficients $(\log B B)$.

\section{References}

I. Jäger R, Schmidt F, Schilling B, Brickmann J: J Comput.-Aided Mol Design 2000, 14:63I.

2. Jäger R, Kast SM, Brickmann J: J Chem Inf Comput. Sci 2003, 43:237.

3. Goodford PJ: Chemometrics 1996, 10:107. 